\title{
Aguardente de Cajuzinho do Cerrado: Produção e Análises Físicas e Químicas
}

\author{
Maria A. B. Gonçalves, Wagner R. Carvalho, Clarissa Damiani, \\ Flávio A. Silva, Márcio Caliari, Yasmini P. A. Silva, Lara K. R. \\ Estevam, Juliana F. Migotto \& Nathalia S. R. Mendes
}

O objetivo deste trabalho foi produzir aguardente de cajuzinho do cerrado e avaliar suas características físicas e químicas. A aguardente obtida foi analisada quanto ao grau alcoólico, densidade, $\mathrm{pH}$, extrato seco, acidez volátil, acidez total titulável, acidez fixa e a quantidade de antioxidantes e compostos fenólicos. A aguardente apresentou compostos antioxidantes nos extratos alcoólicos, oriundos do fruto in natura, melhorando a qualidade nutricional da mesma. Os resultados encontrados ficaram dentro dos parâmetros exigidos pela legislação brasileira e permitem concluir que o cajuzinho do cerrado apresenta potencial para ser utilizado como matéria-prima para produção de aguardente de qualidade.

Palavras-chave: aguardente, Anacardium othonianum Rizzini, antioxidante.

The objective of this work was to produce a "cajuzinho do cerrado" spiriti and to assess their physical and chemical characteristics in terms of alcohol content, density, $\mathrm{pH}$, solids, volatile acidity, total acidity, fixed acidity and the amount of antioxidant and phenolic compounds. The spiriti showed antioxidant compounds in alcoholic extracts, which were obtained from the fresh fruit, suggesting an improved nutritional quality of the beverage. The results were within the parameters required by Brazilian law, allowing to conclude that the "cajuzinho do cerrado" fruit has a potential application as substrate for the production of an improved quality spiriti.

Keywords: spiriti, Anacardium othonianum Rizzini, antioxidant. 


\section{Introdução}

As fruteiras nativas ocupam lugar de destaque no ecossistema do cerrado e seus frutos são comercializados em feiras e com grande aceitação popular. Esses frutos apresentam sabores sui generis e elevados teores de açúcares, proteínas, vitaminas e sais minerais, e podem ser consumidos in natura ou na forma de sucos, licores, sorvetes e geléias, por exemplo. Hoje existem mais de 58 espécies de frutas nativas do cerrado, conhecidas e utilizadas pela população ${ }^{1}$ e, dentre elas, destaca-se o caju do cerrado (Anacardium othonianum Rizzini). A parte carnosa do caju, que é o pseudofruto ou pedúnculo, é muito apreciada no Brasil para consumo in natura ou processado (bebidas, doces, sorvetes e pratos salgados) pelo sabor especial e pelo alto valor nutritivo, relacionado, principalmente, ao elevado teor de vitamina C. A rápida deterioração do pedúnculo de caju é um problema que exige grande atenção, visando alternativas de aproveitamento no campo e na indústria ${ }^{2}$.

A produção anual do caju no Brasil é cerca de um milhão de toneladas, sendo a região nordeste responsável por, aproximadamente, 99\% desta produção. Entretanto, 85\% da produção é desperdiçada, uma vez que o interesse comercial desta fruta está na industrialização da castanha para produção de óleos e castanha comestível, principalmente para exportação ${ }^{3}$.

A utilização do pedúnculo de caju para produção de fermentado (vinho), vinagre e destilado do fermentado (aguardente), entre outros, é uma forma de reduzir este desperdício, aproveitando a parte suculenta e fazendo com que a cultura de caju seja mais valorizada, gerando emprego e renda para os produtores do fruto.

O cajueiro é uma planta rústica, típica de regiões de clima tropical. Na amazônia tropical, as árvores apresentam porte bastante elevado; nos estados do nordeste brasileiro, a principal espécie de ocorrência é o Anacardium occidentale L., cujas árvores apresentam pequeno e médio porte. Nas regiões de cerrado do Brasil Central, as espécies nativas podem apresentar porte médio, como o cajueiro arbóreo-do-cerrado (Anacardium othonianum), porte arbustivo, como o cajueiro-do-campo (Anacardium humile) ou até porte rasteiro (Anacardium nanum e Anacardium corymbosum). As espécies do cerrado produzem pseudofrutos aromáticos, conhecidos como cajuí, caju-do-campo, cajuzinho-do-campo, cajudo-cerrado e caju-rasteiro, caju-de-árvore-do-cerrado, que possuem sabor muito agradável e tamanho bem menor do que o caju produzido no Nordeste. O A. occidentale L. é a única espécie do gênero que é cultivada com finalidade comercial. As demais espécies são exploradas apenas por extrativismo.

O caju nativo no cerrado brasileiro é largamente consumido ao natural ou mesmo sob a forma de sucos, doces e geléias. O pequeno tamanho destes pedúnculos favorece a produção das famosas compotas e desidratados, também conhecidos como passas de caju. A referência sensorial e nutricional da amêndoa e da polpa suculenta faz desta uma das frutas nativas de maior potencial para a exploração sustentada no território brasileiro. Por fermentação, fornece uma espécie de vinho ou aguardente, conhecido por comunidades indígenas como cauim² ${ }^{2}$.

De acordo com a legislação brasileira ${ }^{4}$, brandy de fruta ou aguardente de fruta é a bebida com graduação alcoólica de 36 a $54 \%$ em volume, a $20{ }^{\circ} \mathrm{C}$, obtida do destilado alcoólico simples da fruta, ou pela destilação do mosto fermentado da fruta. O coeficiente de congêneres não poderá ser inferior a $200 \mathrm{mg}$ por $100 \mathrm{~mL}$ em álcool anidro e nem superior a $650 \mathrm{mg}$ por $100 \mathrm{~mL}$ em álcool anidro. O objetivo deste trabalho foi elaborar e caracterizar física e quimicamente a aguardente de cajuzinho-do-cerrado, a fim de se agregar valor a este fruto tão apreciado no cerrado.

\section{MATERIAL E MÉTODOS}

A matéria-prima utilizada para a produção da aguardente foram pedúnculos de cajuzinho do cerrado (Anacardium othonianum Rizzini), adquiridos no cerrado goiano.

Para a produção da aguardente de cajuzinho do cerrado, utilizou-se o pseudofruto carnoso, extraindose a castanha para outros fins. Os pseudofrutos foram sanitizados com solução de cloro a 100 ppm e, então, triturados com água, sendo o suco filtrado. Adicionouse água destilada até obtenção de 2 L de suco, com o teor de sólidos solúveis de $5^{\circ}$ Brix. Em seguida, fezse a suplementação do suco, acrescentando-se 12 g de farelo de trigo, $12 \mathrm{~g}$ de fubá de milho e $60 \mathrm{~g}$ de fermento biológico seco instantâneo (Saccharomyces cerevisiae), 
formando, assim, o pé-de-cuba. Este foi, então, incubado à temperatura ambiente por $24 \mathrm{~h}$. Após este período, adicionou-se $8 \mathrm{~L}$ do suco de cajuzinho ao pé-de-cuba e ajustou-se o teor de sólidos solúveis para $15^{\circ}$ Brix, com a adição de $250 \mathrm{~g}$ de sacarose, iniciando o processo fermentativo para obtenção do álcool etílico, deixando fermentar por $24 \mathrm{~h}$. Ao final, destilou-se o fermentado, extraindo-se cabeça, coração e cauda. O coração foi utilizado nas análises físico-químicas e análise da capacidade antioxidante e presença de fenólicos totais.

\section{ANÁLISES FÍSICO-QUÍMICAS}

As análises físico-químicas foram realizadas em triplicata, conforme metodologia descrita no Manual de Análises de Bebidas e Vinagres do Laboratório Nacional de Referência Vegetal do Ministério da Agricultura, Pecuária e Abastecimento ${ }^{5}$.

- Grau alcoólico real: determinado em função da densidade, a $20^{\circ} \mathrm{C}$, do destilado da amostra, utilizando-se tabela específica

- Densidade relativa $\mathbf{2 0}^{\circ} / \mathbf{2 0}^{\circ}$ : realizada por meio do método densimétrico.

- pH: quantificado em potenciômetro, marca Instrutemp.

- Extrto seco total: quantificado mediante a diferença de massa das placas de petri de aço inóx, antes e após a evaporação da amostra em banho-maria a $100^{\circ} \mathrm{C}$.

- Acidez total titulável: determinada por titulação da amostra com $\mathrm{NaOH}$ 0,1 M, utilizando fenolftaleína com indicador.

- Acidez volátil: determinada pela destilação da amostra por arraste de vapor, seguida de titulação;

- Acidez fixa: determinada pela diferença entre acidez total e a acidez volátil.

\section{TESTES DE ATIVIDADE ANTIOXIDANTE IN VITRO}

Os extratos etéreo, alcoólico e aquoso foram submetidos ao teste do 1,1 difenil-2-picrilhidrazil (DPPH - Marca Sigma), onde a capacidade das amostras de seqüestrar radicais livres foi medida, utilizandose o método descrito por Brand-William et al. ${ }^{6}$, com modificações. Este método baseia-se na remoção do radical estável DPPH do meio de reação pela ação dos antioxidantes presentes na amostra. O grau de descoloração do radical DPPH, a 517 nm, após a adição da amostra, foi medido espectrofotometricamente em uma solução metanólica até a absorbância permanecer constante e indicar a eficiência da amostra adicionada em remover o radical.

A atividade de seqüestro do radical DPPH foi calculada pela Equação (1).

$\%$ DesDPPH $=[1-(\mathrm{AbsA}-\mathrm{AbsB})] \times 100 \mathrm{AbsE}$

Sendo: \% Des DPPH = porcentagem de descoloração do DPPH; Abs A = Absorbância da amostra; Abs B = Absorbância do branco da amostra; Abs E = Absorbância do branco do ensaio do DPPH.

\section{TESTES DE FENÓLICOS TOTAIS}

A presença de fenólicos totais foi analisada, seguindo a metodologia descrita por Borguini ${ }^{7}$. Dos extratos alcoólico e aquoso, anteriormente citados, foram transferidas alíquotas de 0,25 $\mathrm{mL}$ para tubos de ensaio. Nos tubos foram adicionados $0,25 \mathrm{~mL}$ de reativo de Folin Ciocalteau, 0,25 mL de solução saturada de carbonato de sódio e 1,5 mL de água destilada; em seguida, os tubos foram colocados em banho maria a $37^{\circ} \mathrm{C}$ por 30 minutos. Absorbâncias de cada amostra foram determinadas em espectrofotômetro, no comprimento de onda de $750 \mathrm{~nm}$, utilizando água como branco. Todas as análises foram realizadas em duplicata.

\section{Resultados e discussão}

\section{ANÁLISES FÍSICO-QUÍMICAS}

A Tabela 1 apresenta os resultados das análises físico-químicas da aguardente produzida a partir do pseudofruto do cajuzinho do cerrado. O grau alcoólico da aguardente de cajuzinho do cerrado $\left(39,2{ }^{\circ} \mathrm{GL}\right.$ a $20^{\circ} \mathrm{C}$ ) está dentro da faixa estabelecida pela legislação para aguardente de frutas que é de 36 a $54{ }^{\circ} \mathrm{GL}^{8}$. Medina ${ }^{9}$ obteve o valor de $53,08^{\circ} \mathrm{GL}$ para a aguardente de caju. Esse fato deve-se ao maior tempo de destilação no presente trabalho. 
Tabela1.Análisesfísico-químicasdaaguardentedecajuzinho-do-cerrado

\begin{tabular}{|c|c|}
\hline Análise & $\begin{array}{c}\text { Resultado (média } \\
\pm \text { desvio padrão) }\end{array}$ \\
\hline Grau Alcoólico Real $\left({ }^{\circ} \mathrm{GL}\right)$ & $39,2 \pm 0,1$ \\
\hline Densidade $(\mathrm{g} / \mathrm{mL})$ & $0,9427 \pm 0,0003$ \\
\hline Extrato Seco $(\mathrm{g} / \mathrm{L})$ & $0,0667 \pm 0,0170$ \\
\hline $\mathrm{pH}$ & $4,93 \pm 0,01$ \\
\hline $\begin{array}{c}\text { Acidez total } \\
\text { (g ácido acético/100 } \mathrm{mL} \text { amostra) }\end{array}$ & $0,01334 \pm 0,00000$ \\
\hline $\begin{array}{c}\text { Acidez volátil } \\
\text { (g ácido acético/100 } \mathrm{mL} \text { amostra) }\end{array}$ & $0,01251 \pm 0,00000$ \\
\hline $\begin{array}{c}\text { Acidez fixa } \\
\text { (g ácido acético/100 } \mathrm{mL} \text { amostra) }\end{array}$ & $0,00083 \pm 0,00000$ \\
\hline
\end{tabular}

Em relação à densidade, o valor obtido para a aguardente do cajuzinho do cerrado foi de 0,9427 g/ $\mathrm{ml}$. Este valor é próximo ao obtido por Asquieri et al. ${ }^{10}$ para aguardente de jabuticaba $(0,95 \mathrm{~g} / \mathrm{mL})$; por Silva et al. ${ }^{11}$ para aguardente de polpa de banana $(0,952 \mathrm{~g} / \mathrm{mL}$ a 20oC) e aguardente de banana integral (polpa e casca) $(0,949 \mathrm{~g} / \mathrm{mL})$ e por Medina $^{9}$ para aguardente de caju $\left(0,93 \mathrm{~g} / \mathrm{mL}\right.$ a $\left.15^{\circ} \mathrm{C}\right)$.

O valor do extrato seco obtido para a aguardente de cajuzinho do cerrado $(0,0667 \mathrm{~g} / \mathrm{L})$ foi próximo ao obtido por Medina ${ }^{9}$ para aguardente de caju $(0,056 \mathrm{~g} / \mathrm{L})$; inferior ao de Asquieri et al. ${ }^{10}$ para aguardente de jabuticaba $(0,11$ $\mathrm{g} / \mathrm{L})$, e o dobro (0,03 g/L) do valor obtido por Bizelli et al. ${ }^{12}$ para aguardente de cana não envelhecida monodestilada.

$\mathrm{O} \mathrm{pH}$ da aguardente de cajuzinho do cerrado $(4,93)$ foi superior ao obtido por Asquieri et al. ${ }^{10}$ para aguardente de jabuticaba $(2,83)$ e próximo ao obtido por Medina $^{9}$ para aguardente de caju $(4,82)$. A acidez total da aguardente de cajuzinho foi de 13,34 mg/100 mL, enquanto Asquieri et al. ${ }^{10}$ obteve $55 \mathrm{mg} / 100 \mathrm{~mL}$ para aguardente de jabuticaba. De acordo com Bizelli et al. ${ }^{12}$, a acidez pode ser reduzida através de bidestilação e, em seu estudo para acidez total, obtiveram 43,95 mg/100 mL para aguardente monodestilada e 17,95 mg/100 mL para aguardente bidestilada.

A acidez fixa determina-se pela diferença entre acidez total e a volátil. O valor para a aguardente do cajuzinho do cerrado foi de $0,83 \mathrm{mg} / 100 \mathrm{~mL}$ e, mesmo sem passar pelo processo de maturação, este resultado está dentro da faixa quando comparado com os obtidos por Martínez et al..$^{13}$ em aguardentes após 24 horas de maturação. A acidez fixa oscilou entre 0,72 e $8,16 \mathrm{mg} / 100 \mathrm{~mL}$.
De acordo com a legislação brasileira ${ }^{8}$, o valor máximo permitido para a acidez volátil é de $100 \mathrm{mg}$ de ácido acético $/ 100 \mathrm{~mL}$ de amostra e o valor obtido para o cajuzinho do cerrado foi de 12,51 mg de ácido acético/100 mL de amostra. Valores elevados de ácidos voláteis podem ser devido à contaminação microbiana, proveniente da falta de assepsia no processo e a um não recolhimento da fração ideal de destilado ${ }^{14}$, o que comprova a boa qualidade do produto elaborado.

Os resultados das análises antioxidantes da aguardente de cajuzinho do cerrado estão representados na Tabela 2. A atividade antioxidante é uma medida da capacidade das substâncias extraídas da matriz do alimento de seqüestrar radicais livres. Para a aguardente de cajuzinho do cerrado, esta capacidade não foi encontrada nos extratos etéreo e aquoso, enquanto que no extrato alcoólico obteve-se um IC50 de 1408,33 mg/ g de amostra, com descoloração do DPPH no extrato alcoólico de 7,1\%.

Tabela 2. Resultado das análises antioxidantes na aguardente de cajuzinho do cerrado.

\begin{tabular}{|c|c|c|}
\hline Potencial Antioxidante & \% descoloração & $\begin{array}{c}\text { IC 50* } \\
\text { (mg/ g amostra) }\end{array}$ \\
\hline - Extrato Etéreo & 0,0 & 0,00 \\
\hline - Extrato Alcoólico & 7,1 & 1408,33 \\
\hline - Extrato Aquoso & 0,0 & 0,00 \\
\hline $\begin{array}{c}\text { Compostos } \\
\text { Fenólicos Totais (mg } \\
\text { EAG**/100g) }\end{array}$ & \multicolumn{2}{|c|}{9,6263} \\
\hline
\end{tabular}

* $\mathrm{IC}_{50}$ corresponde a quantidade de substâncias com poder antioxidante capaz de reduzir o reagente de DPPH em 50\%; **EAG -Equivalente de ácido gálico.

Quantitativamente foi encontrado na aguardente de cajuzinho 9,6263 (mg EAG/100g) de compostos fenólicos, encontrado apenas no extrato alcoólico. Broinizi et al. ${ }^{15}$ obtiveram, para o bagaço de caju no extrato aquoso, até 10,4 mg de ácido gálico/g bagaço de caju, e no extrato alcoólico, até $2,3 \mathrm{mg}$ de ácido gálico/g bagaço de caju. Portanto, pode-se observar uma maior presença destes compostos no bagaço de caju do que na aguardente de cajuzinho do cerrado. Tal fato deve-se ao processamento empregado na fabricação de aguardente, uma vez que o processo de destilação arrasta apenas uma parte dos compostos fenólicos para a aguardente, mas ainda assim, colaborando com a qualidade nutricional do produto final. 


\section{Conclusões}

Pela análise dos resultados obtidos, constatou-se a viabilidade de se utilizar o cajuzinho do cerrado como matéria-prima para a produção de aguardente, pois se obteve um produto com características físico-químicas dentro dos parâmetros estabelecidos na legislação brasileira. Além disso, a produção da aguardente de cajuzinho do cerrado pode ser uma maneira de despertar o interesse e disseminar o conhecimento sobre os frutos do cerrado brasileiro.

Observou-se, também, que a aguardente de cajuzinho do cerrado possui características particulares em relação à capacidade antioxidante, o que agregou valor a este produto, tornando-o uma alternativa plausível para a agroindústria de frutos do cerrado.

\section{Referências}

1. Silva, A. P. P., Melo, B., Fernandes, N. Fruteiras do cerrado. Núcleo de Estudos em Fruticultura no Cerrado, p. 1, 2003. em: <http://www.fruticultura.iciag.ufu.br/fruteiras\%20do\%20 cerrado>. Acessado em dez. 2009.

2. Costa, T. S. A.; Faria, J. P.; Naves, R. V.; Vieira, R. F.; Costa, T. S. A.; Da Silva, D. B.; Ferreira, F. B.; Sano, S. M.; Embrapa recursos genéticos e biotecnologia, 2006, cap. 8.

3. Neto, A.B.T.; Silva, M. E.; Silva, W. B.; Swarnakar, R.; Silva, F. L. H.; Quím. Nova 2006, 29(3).

4. Brasil. Decreto $n^{0} 2.314$, de 04 de setembro de 1997. Normas gerais sobre registro, padronização, classificação e inspeção e fiscalização da produção e do comércio de bebidas, 1997.

5. Brasil. Ministério da Agricultura, Pecuária e Abastecimento. Instrução Normativa $n^{\circ}$ 24, de 08/09/2005. 2005. Aprova o Manual Operacional de Bebidas e Vinagres. Manual de Métodos de Análise de Bebidas e Vinagres. Caderno 5: Destilados. em: <http://extranet. agricultura.gov.br/sislegis-consulta/consultarLegislacao.do? operacao=visualizar\&id=13576> . Acessado em out. 2009.

6. Brand-William W.; Cuvelier, M. E.; Berset, C.; Lebensmwiss. U-Technology 1995, cap. 28.

7. Borguini, R. G.; Tese de Doutorado, Universidade de São Paulo, Brasil 2006.
8. Brasil. Ministério da Agricultura, Pecuária e Abastecimento. Portaria $n^{\circ}$ 65, de 23 de abril de 2008: regulamento técnico para a fixação dos padrões de identidade e qualidade para aguardente de fruta. Brasília, DF, 2008. em <www. agricultura.gov.br>. Acessado em set. 2009.

9. Medina, J. C.; University of Chicago Press, 1978, 4, 143.

10. Asquieri, E. R.; Moura, A. G. S.; Cândido, M. A.; Ciência e Tecnologia de Alimentos. 2009, 29(4), 896.

11. Silva, M. B. L.; Chaves, J. B. P.; Lelis, V. G., Alvarenga, L. M.; Zuim, D. R.; Silva, P. H. A.; Alim. Nutr. 2009, 20(2), 217.

12. Bizelli, L. C.; Ribeiro, C. A. F.; Novaes, F. V. Scientia Agrícola, 2000, 57(4), 623.

13. Martínez, R. G.; Serrana, L. G.; Mir, M.V.; Martínez, M. C. L. Alimentaria 1997, 284, 111.

14. Vargas, E. A.; Gloria, M. B.; Ciência e Tecnologia de Alimentos, 1995, 15(1), 43.

15. Broinizi, P. R. B.; Andrade-Wartha, E. R. S.; Silva, A. M. O.; Torres, R. P.; Azeredo, H. M. C.; Alves, R. E.; Mancini-Filho, J.; Ciência e Tecnologia de Alimentos, 2007, 27(4), 902.

\section{Maria A. B. Gonçalves*, Wagner R. Carvalho, Clarissa Damiani, Flávio A. Silva, Márcio Caliari, Yasmini P. A. Silva, Lara K. R. Estevam, Juliana F. Migotto \& Nathalia S. R. Mendes.}

${ }^{1}$ Universidade Federal de Goiás, Campus Samambaia - Rodovia Goiânia / Nova Veneza, Km 0 - Caixa Postal 131 - CEP 74001-970 - Goiânia - GO.

*e-mail: assima.bittar@gmail.com 